\title{
A Food Management Based on Smart Refrigerator System
}

\author{
R. Sudha ${ }^{1}$ and G. Indirani ${ }^{2}$ \\ ${ }^{1}$ Research Scholar, ${ }^{2}$ Assistant Professor \\ ${ }^{1 \& 2}$ Department of Computer Science and Engineering, Annamalai University, Tamil Nadu, India \\ E-Mail: sudharajcse@gmail.com, Induk0992@gmail.com
}

\begin{abstract}
Internet of Things (IoT) conceptualizes the idea of remotely connecting and monitoring real world objects (things) through the Internet [1]. When it comes to our house, this concept can be aptly incorporated to make it smarter, safer and automated. This IoT paper focuses on smart refrigerator system which sends alerts to the user when the milk packets count to be decreased because the user refilling he packet purpose. The advantage of smart system is to maintaining the performance and avoids unavailability of milk. Besides, the same can also be utilized for smart fridge by making use of the sensors. The leverage obtained by preferring this system over the similar kinds of existing systems is that the alerts and the status sent by the wifi connected microcontroller managed system can be received by the user on his phone from any distance irrespective of whether his mobile phone. The microcontroller used in the current prototype which comes with an embedded micro-controller and an onboard Wi-Fi shield making use of which all the elctrical appliances inside the home can be controlled and managed.

Keywords: Refrigerator, IoT (Internet of Things), Load Cell Sensor, Arudino UNO, Threshold
\end{abstract}

\section{INTRODUCTION}

Refrigerator is the most frequently used domiciliary/kitchen electrical appliance all over the world for food sarduiotorage. Principally this appliance is used for various tenacities like storing vegetables, fruits etc. Smart refrigeration module is designed to transfigure any existing refrigerator into a smart cost effective machine using sensors. Smart refrigerator compares the status of the food for e.g. expiry date, weight, quantity etc. Significance of this work will be removable of food spoilage, reduce illness and make healthier lifestyle of modern age human being. Smart applications with hypermedia capability are being used in today's life, all the major credit goes to digitalization of technology and wide usage of internet. In this modern era, human being is used to deal with technology or we can say it as internet of things (IoT).

As we look around ourselves we see modernization with superior technology, for example cell phones, kitchen, appliances and many more. Smart appliances include washing machine, television, refrigerator etc. Here we study about smart refrigerator, because people are very busy in modern lifestyle. They do not really have time to look after their basic healthy habits and diet; since we are capable to deal with the technology we can design a smart refrigerator system which can help us to maintain a healthier lifestyle without putting any extra effort and time. In this paper we propose smart refrigerator which leads to healthier lifestyle. Smart refrigerator is designed for managing food items stored in it and advising it user what type of food store inside the refrigerator. Here we discover the presence of the object by using load cell sensor, which is used for checking the weight of products of the container where objects are placed. It is always challenging to develop smart appliances while we appreciate about a smart home.

A smart refrigerator is the main motivation while we talk about our research. We have seen much advancement while developing smart refrigerator in industry and research. The industry tries to change the generalized refrigerator function, i.e. to store food items in a suitable environment these computer-operated capabilities allow the development of applications for many devices one of them is smart refrigerators. In this paper, we propose to develop smart refrigerator which is an intelligent embedded system and allows the user to manage and accurately locate the milk packets stored inside it. Similar number of milk packets is placed at different-different blocks.

It is a fact that the fast-paced development and today's modern living has brought a depressive change on people' lifestyle towards less physical activities and efforts and an uneven poor maintenance. Careless counting can cause shortage of counting. For instance, problems resulting from unavailability of milk packets and lifestyles are becoming a big health threat in the most of the developed nations. In this index we believe that applications which targets on proper maintenance are important. Our work will be to present an application development which will mainly focus towards the smart refrigerator with better performance.

\section{LITERATURE SURVEY}

\section{A. Smart Refrigerator Using Internet of Things}

The Smart Refrigerator system is mainly implemented to convert the existing refrigerator into an intelligent cost effective appliance .The smart refrigerator system is capable of sensing as well as monitoring its contents. The smart refrigerator system is able to remotely notify the user about the scarce products via SMS and email .The Monitoring unit of the system has a software module in its memory, which will be always in the monitoring mode and will scans its input. 


\section{B. Energy Efficient Smart Home Automation System}

The high energy needed by home Appliances (like white goods, audio/video devices and communication equipments) and Air-con systems (heating and cooling), makes our homes one among the foremost essential areas for the impact of energy consumption on natural surroundings .AIM for the planning of a system which will minimize energy waste in home environments with efficiency managing the devices operation modes. In our design we tend to use a wireless design elements to observe the physical parameters (like light-weight and temperature) additionally because the presence of users reception and in every of its rooms. In order to optimize the energy consumption and value while guaranteeing the specified comfort level. When users change their habits as a result of unpredictable events, the system is able to notice wrong predictions analyzing in real time info from sensors and to switch system behavior consequently. The parameters are might to stop the introduction of home automation systems for energy saving into the mass market.

\section{Multiple Appliances Controlling and Monitoring System based on Wireless Embedded Home Gateway}

In this paper, the author presents a prototype with the low cost wireless embedded gateway for remote Controlled home and also monitoring other connected systems through internet. The gateway in the proposed prototype provides the data transfer between the user and multiple number of home appliances through connection of internet. The light weight Constrained Application Protocol (CoAP) is used to provide better and efficient data transfer between the gateway and the Wireless Sensor and Actuator Modules (WSAM). This system mainly uses wireless technology to avoid connection of wire between appliances and the gateway.

It helps to do complete monitoring and control functionalities of the home appliance environment using wireless sensors and actuators modules than just the switching operation like ON/OFF functionality provided by other similar systems. By using the proposed prototype system in the home, appliances can be automated at lower cost. This system does not require any dedicated expensive components which includes a server PC but as it uses only low cost embedded devices.

\section{Smart Home Electricity Management System Using Cloud Computing}

This paper proposes a system of home automation using Cloud computing. The system consists of collecting on-line data for power consumption and then manipulating the power supply of connected electrical devices. Along with controlling of electrical devices, this system can generate reports on daily, monthly or yearly basis of cost or usage of electricity of each appliance connected to the system.

\section{E. Bluetooth Based Wireless Home Automation System Using FPGA}

This paper proposes a Home Automation system which is controlled by using Android smart phone. Bluetooth module (HC-05) is being used to control different home appliances which are connected to the input and output pins of FPGA board and a serial communication between Bluetooth module and FPGA board is carried out. Bluetooth provides a simple and efficient communication path between different peripherals. HC-05 is comparatively a better module as it can easily switch between its master and slave mode and also after disconnecting from the peripheral it will start searching for other Bluetooth devices without any prerequisite command. Provided home automation system is secured and flexible and is controlled using an android application in a mobile phone. FPGA board is used to control the electrical devices which are connected to FPGA board through relay. A wireless communication between Bluetooth module and android phone is established. That module is serially connected to the FPGA board through RS-232 for the controlling of home appliances. The whole system is controlled by using either AT commands or using the switches provided in the android application.

\section{PROPOSED SYSTEM}

The proposed design aims to implement a smart refrigerator system, which is easy to use and economical for the user. It is capable of notifying the owner about their activities going on inside it via wireless system on the mobile phone. The Internet of things based systems where they will be able to see the condition of the number of milk packets kept inside the refrigerator. The whole system is governed by the Arudino UNO microcontroller where load sensor cell act as an input of microcontroller and Wi-Fi transmits all information to the wireless sensors by using IoT. The items weight is below the set threshold value to alert notification is sent to the user's mobile to refill the milk packets before the get over.

\section{SYSTEM ARCHITECTURE}

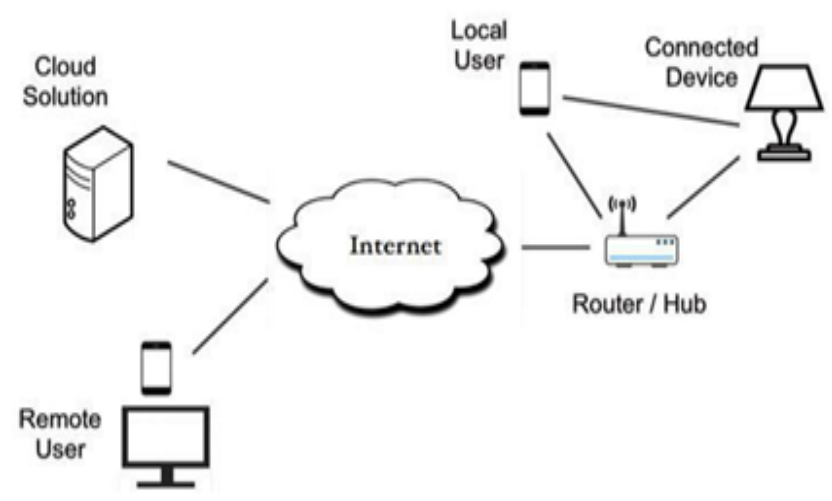

Fig. 1 Block Diagram 


\section{PROCESS FLOW}

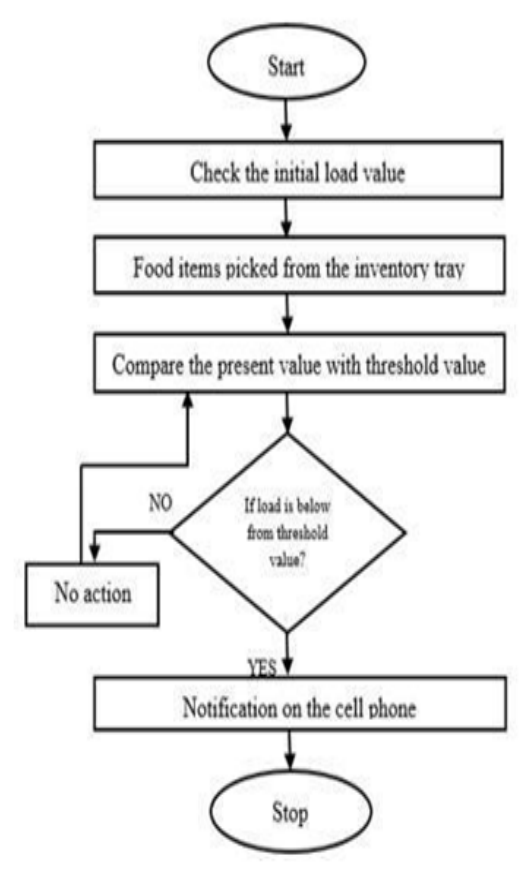

Fig. 2 Process Flow

The steps followed for designing the system are:

1. Check the initial value of the number of packets which is kept inside the refrigerator

2. No of Packets picked from the inventory tray

3. Compare the status of taken packets (present value with threshold value)

4. If load value is below from threshold value then send the notification to the user on the mobile phone.

The system comprises of many sections where the sensors are placed. A load cell is a transducer that is used to create an electrical signal whose magnitude is directly proportional to the force being measured. This electronic signal can be a voltage change, current change or frequency change depending on the type of load cell and circuitry used. The electrical signal output is typically in the order of a few milli-volts. Whenever the food items kept in the refrigerator goes below the present value than it generates an alert which is being transmitted in the form of message to the user. After following the above steps we are now able to get real time information, quantity of food items which are stored inside the refrigerator.

\section{SENSOR IMPLEMENTATION USING ARUDINO} UNO.

A. Load Cell: Over weighting detection in compartment of refrigerator can be detected using load cell.

Load Cell Sensor generally consists of a spring element on which strain gauges have been placed. The spring element is usually made of steel or aluminum. That means it is very sturdy, but also minimally elastic. As the name "spring element" suggests, the steel is slightly deformed under load, but then returns to its starting position, responding elastically to every load. These extremely small changes can be acquired with strain gauges. Then finally the deformation of the strain gauge is interpreted by analysis electronics to determine the weight.

This arduino uses the HX711 load Cell amplifier interface. We are interfacing $5 \mathrm{Kg}$ load cell to the arduino using HX711. Fig. 4 shows Load cell amplifier module. HX711 is a precision 24-bit analog to-digital converter (ADC) designed for weigh scales and industrial control applications to interface directly with a bridge sensor. The input multiplexer selects either Channel A or B differential input to the low-noise programmable gain amplifier (PGA). Channel A can be programmed with a gain of 128 or 64, corresponding to a full-scale differential input voltage of $\pm 20 \mathrm{mV}$ or $\pm 40 \mathrm{mV}$ respectively, when a $5 \mathrm{~V}$ supply is connected to AVDD analog power supply pin. Channel B has a fixed gain of 32. On chip power supply regulator eliminates the need for an external supply regulator to provide analog power for the ADC and the sensor. Clock input is flexible. It can be from an external clock source, a crystal, or the on-chip oscillator that does not require any external component. On-chip power on-reset circuitry simplifies digital interface initialization. There is no programming needed for the internal registers. All controls to the HX711 are through the pins.

\section{B. HX711 Module}

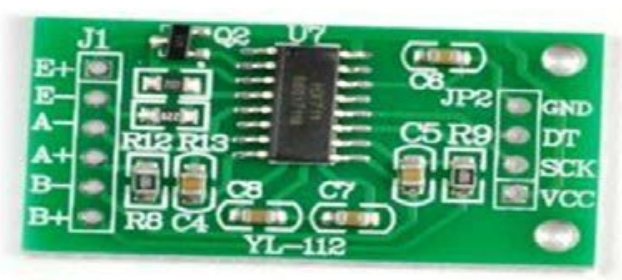

Fig. 3 HX711 Module

The Load cell sensor fig. 4 has four wires red, black, green and white. On HX711 board you will find E+, E-, A+, A- and B+, B-connections. Connect load cell.

1. Red wire to $\mathrm{E}^{+}$

2. Black wire to E-

3. Green wire to A-

4. White wire to $\mathrm{A}^{+}$

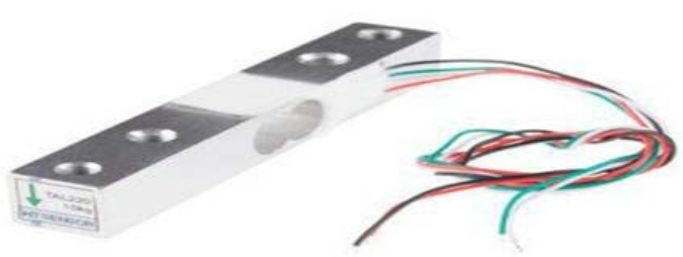

Fig. 4 Load Cell Sensor 


\section{Load Cell connections to HX711 and Arduino: Load Cell} Interface with Arduino

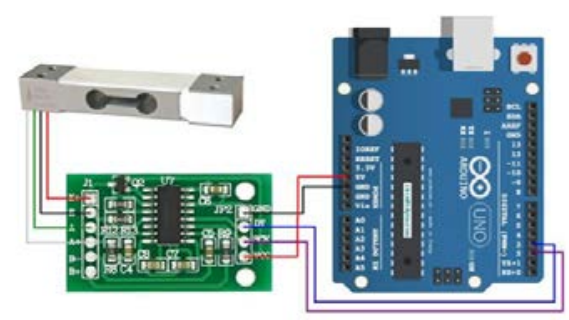

Fig. 5 Connection diagram of the proposed system

Where to apply weight on load cell

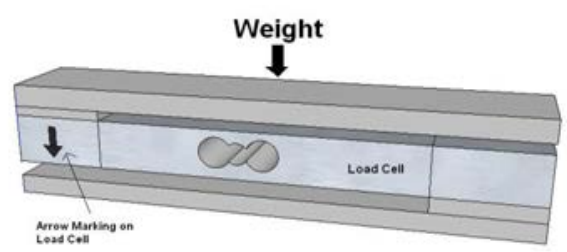

Fig. 6 Load cell sensor weight sketch

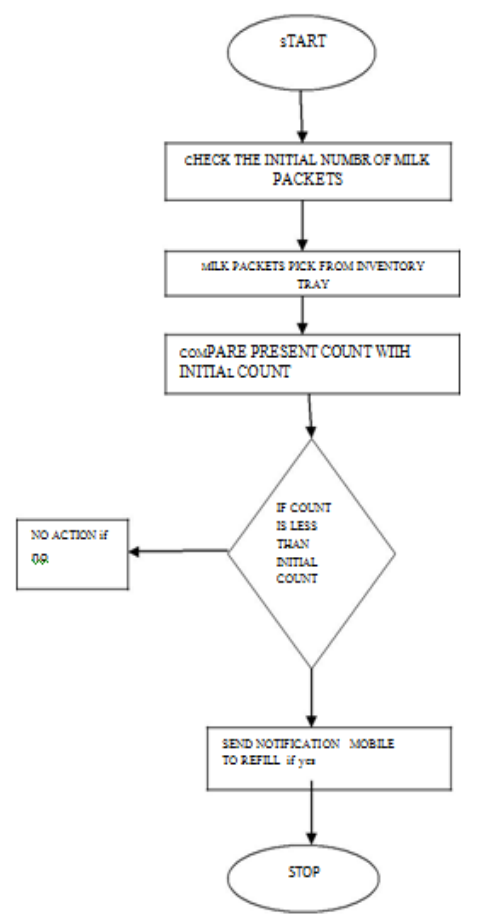

Fig. 7 Block Diagram of Proposed System

Fig. 8 shows the systematic block diagram of Smart Refrigerator using arduino ATMEGA 328p controller board. All the sensors used with their functions are shown in block diagram of fig 7 . We are using sensors like Load cell sensor, GSM Interface; sense respective Parameters and that value will be sent to the Arduino board. Then according to the received values from sensors Arduino will take particular action specified in program stored inside memory of Arduino.

The output devices like mobile used to display warnings to the user as well as warning alert so that user can understand warning from refrigerator. When things go below threshold level that indication will be given to the user's app then user will place the order if necessary.

\section{FUNCTIONAL DESCRIPTION OF REFRIGERATOR}

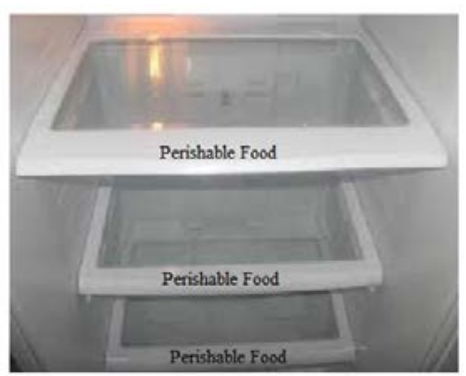

Fig. 8 Functional Description of Refrigerator

Functional descriptions of smart refrigerator are given below.

Perishable food items (particular vegetable or fruits) kept in block first. If vegetable is below from threshold value (200grm) then send the notification on the android app and also press the switch button then start the timer. After some time it will send the notification on the app showing expiry of food items. Another type of food like dairy products and confectionary products kept in block second and third, the functioning is similar as first block.

\section{RESULTS}

Here the snapshots to be attached based on the milk packet .By using the future work we have to implement the system for shopkeeper, Juice and other Medical Appliances. In our proposed work the milk packet is measured by the kilograms. Because the refrigerator contains small amount of food and milk packets. So it will be measured in only kilograms.

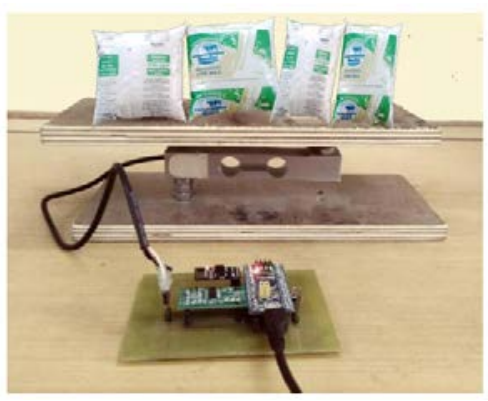

Fig. 9 Hardware Module of Refrigerator 

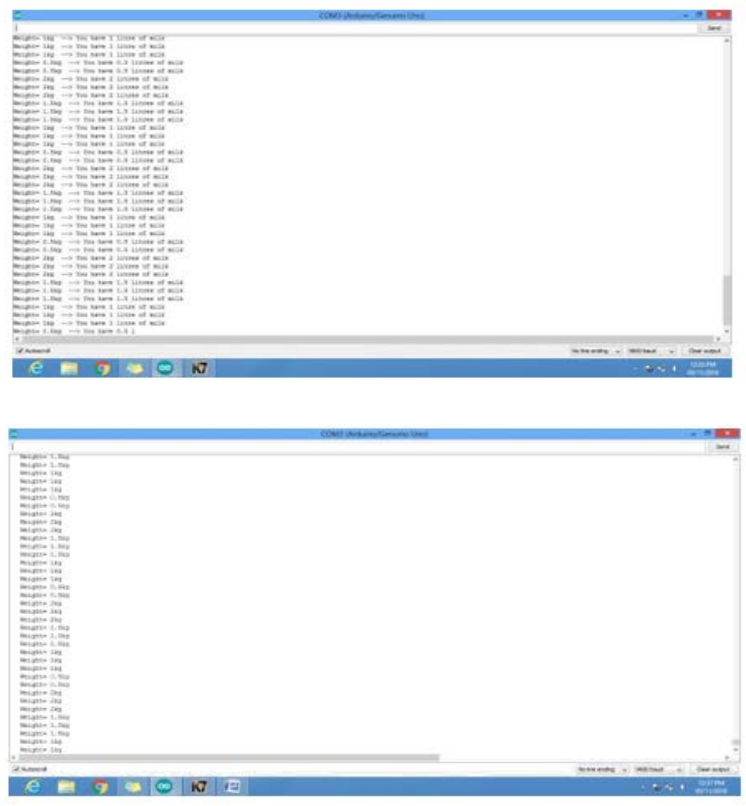

Fig. 10 Calculation of weight by Load cell sensor

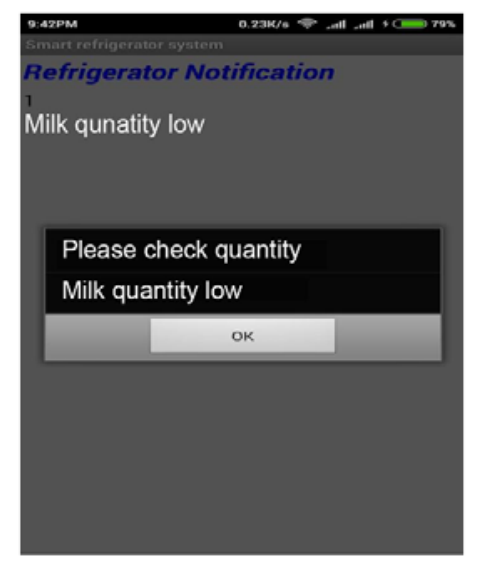

Fig. 11 Notification for Low Quantity of Food Item

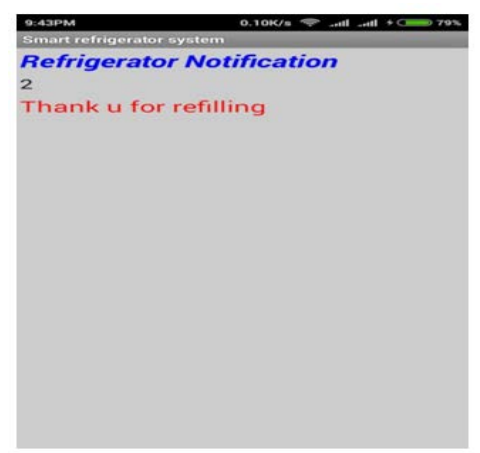

Fig. 12 Notification for Refilling of Content

\section{CONCLUSION}

We have introduced smart refrigerator application with intelligent multimedia capability. The proposed smart refrigerator can measure the count. It is designed for managing items stored in it. More importantly, it can perform other functions such as delay, throughput etc and also through the smart refrigerator people can save money with less effort [2]. We are confident that such type smart refrigerator will be important component in future smart homes. The concept of smart refrigerator is far more reaching than notifying the user about the contents of the refrigerator. Smart refrigerator is cost effective, economical and user friendly.

\section{FUTURE WORK}

The concept of smart refrigerator is far more reaching than notifying the user about the contents of the refrigerator. It should give importance on maintaining milk packet level. If the level is decreased, message is sent to shop keeper. So shopkeeper knows about the milk packet availability. The future smart refrigerator can use the gas sensor to check the freshness of food item and also use the CCTV camera for visualization of all activities inside the refrigerator and display it to the user in real time and to monitor the replacement of food items kept inside it. The refrigerator of the future would then be able to cross reference and act on reducing the ingredients used in future helping to minimize food waste.

\section{REFERENCES}

[1] Mukesh P. Mahajan, Rohit R. Nikam , Vivek P. Patil, and Rahul D. Dond "Smart Refrigerator Using IOT "Vol. 2, No. 3,PP - 8691, March - 2017

[2] S B Prapulla, Dr. G Shobha and Dr. T C Thanuja "Smart Refrigerator Using Internet of Things” Journal of Multidisciplinary Engineering Science and Technology (JMEST), Vol. 2, No. 7, July - 2015.

[3] Nikhil Kakade, and Prof. (Dr.) S. D. Lokhande, "IoT based Intelligent home using Smart Devices", International Journal of Innovative Research in Computer and Communication Engineering, Vol. 4, No. 6, June 2016.

[4] Deepti Singh, and Preet Jain, "IoT Based Smart Refrigerator System”, International Journal of Advanced Research in Electronics and communication Engineering (IJARECE), Vol. 5, No. 7 on -Wireless Information Networks \& Business Information System WINBIS, (2011), July 2016

[5] A.M. Adami, M. Pavel, T.L. Hayes, C.M. Singer, "Detection of Movement in Bed Using Unobtrusive Load Cell Sensors," in Information Technology in Biomedicine, IEEE Transaction, Vol.14, No.2, pp.481-490, March 2010

[6] Lei Xie, Bo Sheng, Yafeng Yin, Sanglu Lu, and Xiang Lu, I Refrigerator: An Intelligent Refrigerator for Food Management based on RFID Technology, Ubi Comp'13, Zurich, Switzerland, September 8-12, 2013.

[7] Deepti Singh, and Preeti Jain, "IoT-based Smart Refrigerator System" International Journal of Advanced Research in Electronics and Communication Engineering (IJARECE) Vol. 5, No. 7, July 2016

[8] Suhuai Luo, Jesse S. Jin, and Jiaming Li, "A Smart Fridge with an Ability to Enhance Health and Enable Better Nutrition” International Journal of Multimedia and Ubiquitous Engineering, Vol. 4, No. 2, April 2009.

[9] Y Zhai, Y Liu, M Yang, F Long, and J Virkki, “A Survey Study of the Usefulness and Concerns about Smart Home Applications” Open Journal of Social Sciences Vol. 2, No. 11, Article ID: 51898,7 pages 10.4236/jss.2014.211017 in 2014

[10] ICT Lee, PAN Fu-Cheng, "Intellectual refrigerator combining With a portable electric device”, United States Patient in 2014

[11] Mukesh P Mahajan, Rohit R Nikam, Vivek P Patil, and Rahul D Dond, "Smart Refrigerator using IoT", International Journal of Latest Engineering Research and Application (IJLERA), Vol. 2, pp. 86-91, 03, March-2017. 